\title{
Antibiotics and Elisa As a Hyphenated Technique for Screening High Production Cell Lines from Transfected HEK293 Cells for the Production of Recombinant Follicle Stimulating Hormone
}

\author{
Jian-wen HU, Meng-meng LIU, Ji-zhong HAN and Bin ZENG ${ }^{a}{ }^{*}$ \\ Jiangxi Province Key Laboratory of Bioprocess Engineering, Jiangxi Science \& \\ Technology Normal University, Nanchang , 330013, China. \\ azengtx001@aliyun.com \\ ${ }^{*}$ Corresponding author
}

Keywords: Recombinant follicle stimulating hormone (rFSH), ELISA, HEK293 cells.

\begin{abstract}
Recombinant follicle stimulating hormone(rFSH), which play an important role in assisted reproductive technologies. However, the availability of a suitable system for the screening high production cell lines from transfected mammal cells for the production of $\mathrm{rFSH}$ is limited. The purpose of this study was to establish a method for screening cloned cell lines with high productivity from transfected HEK293 cells. Here, we adopt the antibiotics in combination with ELISA as a hyphenated technique to obtain production cell lines, and the results indicated that this a simple and efficient method in the cloned cell screening application.
\end{abstract}

\section{Introduction}

Recombinant follicle stimulating hormone(rFSH), which play an important role in assisted reproductive technologies. The currently commercially available rFSH such as Gonal-F, Puregon, Elonva, and Bemfola et al,.were produced by genetic engineering and cell biotechnology[1]. These biopharmaceutical products were produced by mammal cells expression system.

The first step in the manufacture of rFSH is the development of a stable, high expressing recombinant cell lines. However, the availability of a suitable system for the screening high production cell lines from transfected mammal cells for the production of $\mathrm{rFSH}$ is limited. Therefore, it's very necessary to find an simple and effecient method to obtain cloned cell lines with high productivity. Currently, the constuction of efficient expression vector[2], host cell transformation[3], and medium optimization were introduced. Only these strategies are not enough in biopharmaceutical manufacturing, and thus high throughput screening is essential in identification and isolation of high production clones from a heterogeneous population of transfected cells [4].

Here, we adopt the antibiotics in combination with ELISA as a hyphenated technique for obtaining production cell lines from transfected HEK293 cells for the production of rFSH protein with His tags.

\section{Materials and Methods}

\section{Construction of the rFSH Expression Vector}

Genes encoding the FSH $\alpha$-subunit and FSH $\beta$-subunit, including the signal peptide sequences, were constructed by assembly of synthetic oligonucleotides using the 
polymerasechain reaction [5]. The primer pairs contained NheI and BamHI enzyme sites, the upstream primer was designed to carry a Nhel restriction site and the downstream primer carried a BamHI restriction site. The reaction conditions for all reactions were as follows: 35 cycles of denaturation at $94^{\circ} \mathrm{C}$ for 45 second, annealing at $58^{\circ} \mathrm{C}$ for 30 second, and extension at $72^{\circ} \mathrm{C}$ for 40 second. The pIRES-Hygro3 expression vector was obtained from modified pIRES using Hygromycin, and pIRES-Neo3 expression vector was obtained from modified pIRES using Neomycin. The FSH $\alpha$-subunit gene and FSH $\beta$-subunit gene were inserted into PIRES-Hygro3 and pIRES-Neo3 after digestion with NheI and BamH I, respectively.

\section{Expression System and Screening System}

Expression vectors containing the $\alpha$ - and $\beta$-subunit genes were transfected into a HEK293 cell lines. Twenty-four hours after transfection, HEK293 cells were split into cell culture dishes and fed with selection medium. Selective medium formula that DMEM (Hyclone, USA) with 10\% (v/v) fetal bovine serum (WISENT, Canada), 80-100 ug/ml Hygromycin (Roche) and 3000-5000 ug/ml G418 (Invitrogen). The stably expressing $\mathrm{rFSH}$ cloned cell lines were isolated by growth under selection pressure, and this process called antibiotics screening. Subsequently, fresh selection medium was replaced every 3 days and 2 weeks later were picked and seeded in a 24-well plate. When the cell density reach sixty or eighty percent after inoculation, the supernatant was detected by a sandwich-antibody enzyme linked immuno-sorbent assay and the steps as follows. 1) Coating, polystyrene ELISA 96-well microplates (Costar,USA) were coated with 100ul/well of anti-FSH $\alpha$ antibody (Abcam,UK) (5.6ug/mL) used as capture antibody, overnight at $4{ }^{\circ} \mathrm{C}$. Subsequently, the wells were washed (3 4 x $1 \mathrm{~min}$ ) with PBST (pH 7.2-7.4, containing 0.05\% Tween-20). The plate wash was repeated after every step of the assay. 2) Blocking, wells were blocked with $320 \mathrm{ul} /$ well of $5 \%$ skim milk, incubation for $2 \mathrm{~h}$ at $37{ }^{\circ} \mathrm{C}$. 3)Adding samples and set up the controls, add 100ul transfected HEK293 cells supernatant to each well, and simultaneously set up the negative control groups (non-transfected HEK293 cells supernatant) and the blank control groups (medium) were introduced, then incubation for 1 hours at $37{ }^{\circ} \mathrm{C}$. 4) Incubation with secondary antibodies, the His tag antibody (ImmunoWay,USA) diluted 1:16000 in PBS buffer, then add 100ul to each well and avoid light exposure incubation for $1 \mathrm{~h}$ at $37{ }^{\circ} \mathrm{C}$. 5) Adding goat-anti-Rabbit-HRP conjugate, GAR-HRP conjugate (CWBIO,China) was used for signal detection. The antigen-antibody complexes formed were detected via incubation with $100 \mathrm{ul} /$ well of GAR-HRP( diluted 1:4000 in PBST buffer), incubation for 40 minutes at $37{ }^{\circ} \mathrm{C}$. 6) Color development and read $\mathrm{OD}_{450}$ value, the presence of enzyme complexes was detected by the addition of $100 \mathrm{ul} /$ well of TMB reagent (Solarbio, China). The reaction was carried out in complete darkness at $37{ }^{\circ} \mathrm{C}$ for $8-12 \mathrm{~min}$, and was stopped with 50 $\mathrm{ul} /$ well of $2 \mathrm{M}$ sulphuric acid. Absorbances were read after $5 \mathrm{~min}$ at $450 \mathrm{~nm}$, using an automatic microplate reader (Thermo Lab system MK-3,USA).

\section{Collection of Cell Culture Supernatant and SDS-PAGE Analysis}

The media are collected, removal of impurities through a $0.45 \mathrm{um}$ filter devices (Millipore, USA), and further concentrated by the supernatant using filtration through a $15 \mathrm{ml}$ 3KD/UFC9 (Amicon, Millipore, USA) and 6x-His Ni-agarose affinity column(CWBIO, China). The rFSH molecular weight were evaluated by sodium dodecyl sulphate polyacrylamide gel electrophoresis (SDS-PAGE) after denaturation with the sample buffer at $100{ }^{\circ} \mathrm{C}$ for $10 \mathrm{~min}$. The SDS-PAGE separating gel was $12.0 \%$ 
acrylamide in $1.5 \mathrm{M}$ Tris- $\mathrm{HCl}$ buffer of $\mathrm{pH} 8.8$ and stacking gel was $5.0 \%$ acrylamide in $1 \mathrm{M}$ Tris- $\mathrm{HCl}$ buffer of $\mathrm{pH} 6.8$. After electrophoresis, the gels were stained with $0.1 \%$ (w/v) Coomassie blue R-250 and analyzed using the Gel Imaging System (Bio-Rad, USA ) [6].

\section{Results}

\section{Identification of Recombinant Plasmids}

We also used restrict enzyme and electrophoretogram to verify the reconstructed pIRES-Hygro3-FSH $\alpha$ and pIRES-Neo3-FSH $\beta$ plasmid (as show in Fig.1). Electrophoretogram analysis results as follows. (a) In the case of the pIRES-Hygro3-FSH $\alpha$ plasmid, we observed two bands of approximately 6300 and $4000 \mathrm{bp}$, and the electrophoretic migration rate of the ring recombinant plasmid significantly faster than linear recombinant plasmid. The pIRES-Hygro3-FSH $\alpha$ plasmid was digested with NheI and BamHI and resolved via electrophoresis, we observed fractions of most of the expected molecular mass of approximately5700bp. and the ring recombinant plasmid of electrophoretic migration rate significantly faster than linear recombinant plasmid .(b) Similarly, as for the pIRES-Neo3-FSH $\beta$ plasmid, we observed two bands of approximately 6000 and $3500 \mathrm{bp}$, also because the electrophoretic migration rate of the ring recombinant plasmid significantly faster than linear recombinant plasmid . we observed fractions of most of the expected molecular mass of approximately 5000bp.(c) KB ladder. The electrophoretogram analysis results showed that the two expression vector with target gene were constructed successfully. This process was completed by Gen Script Corporation, Nan Jing, Jiangsu province, China.
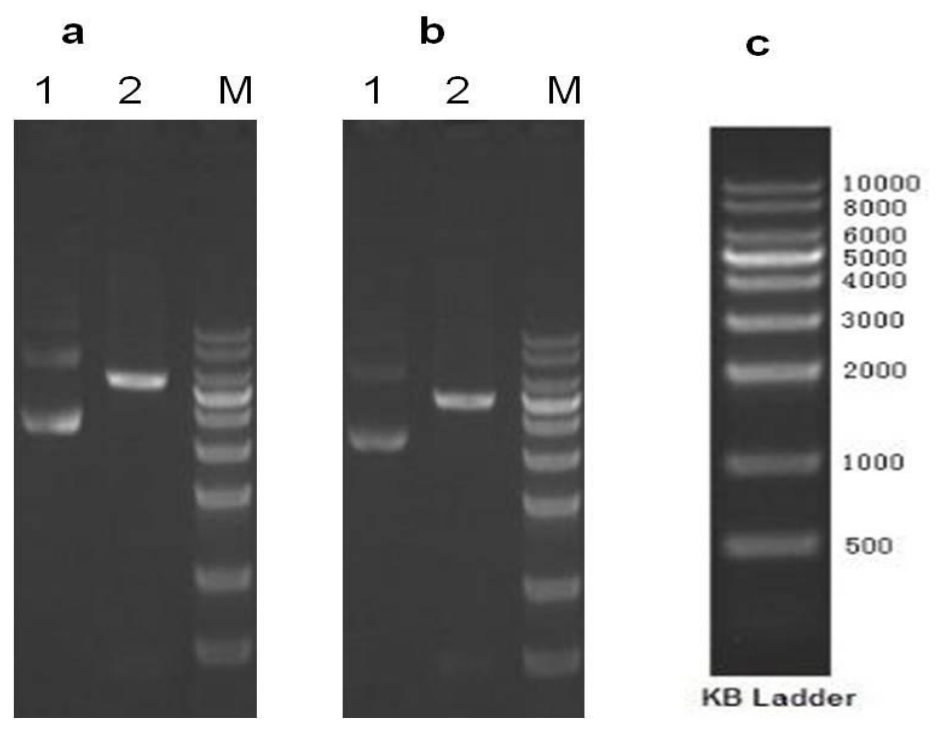

Figure 1. Electrophoretogram analysis results.

(a)pIRES-Hygro3-FSH $\alpha$ plasmid, Lane1:pIRES-Hygro3-FSH $\alpha$ plasmid. Lane2:pIRES-Hygro3-FSH $\alpha$ plasmid digested by NheI and BamHI. Lane M: KB ladder .

(b)pIRES-Neo3-FSH $\beta$ plasmid,Lane1:pIRES-Neo3-FSH $\beta$ plasmid. Lane2: pIRES-Neo3- FSH $\beta$ plasmid digested by NheI and BamHI. Lane M: KB ladder.

(c) KB ladder 


\section{High through Put Screening System}

HEK293 cell are frequently used in biomedical research for heterologous protein expression. After the expression vectors had entered the host cell, we adopt the method of a combination of antibiotics and enzyme-linked immunosorbent assay (ELISA) as a hyphenated technique for the screening high expression of cloned cell lines. Stable clones expressing rFSH were isolated by growth under selection pressure, total treatment length based on the selected concentration of antibiotics, but it typically 2 to 3 weeks(as show in Fig.2). The stable expression of individual clones were further determined by ELISA, the supernatant was detected with double antibody sandwich enzyme linked immunosorbent assay. The $\mathrm{OD}_{450}$ values of experimental groups(cloned cell lines cultuer supernatant) were significant differences compared with the control groups. The $\mathrm{OD}_{450}$ values of negative control group(non-transfected cells cultuer supernatant) and blank control group (medium) almost unchanged (as show in Table 1). The high expression of rFSH cloned cell lines were selected from transfected HEK293 cells based on high $\mathrm{OD}_{450}$ values. All these data confirmed the screening system was sensitive and practice.
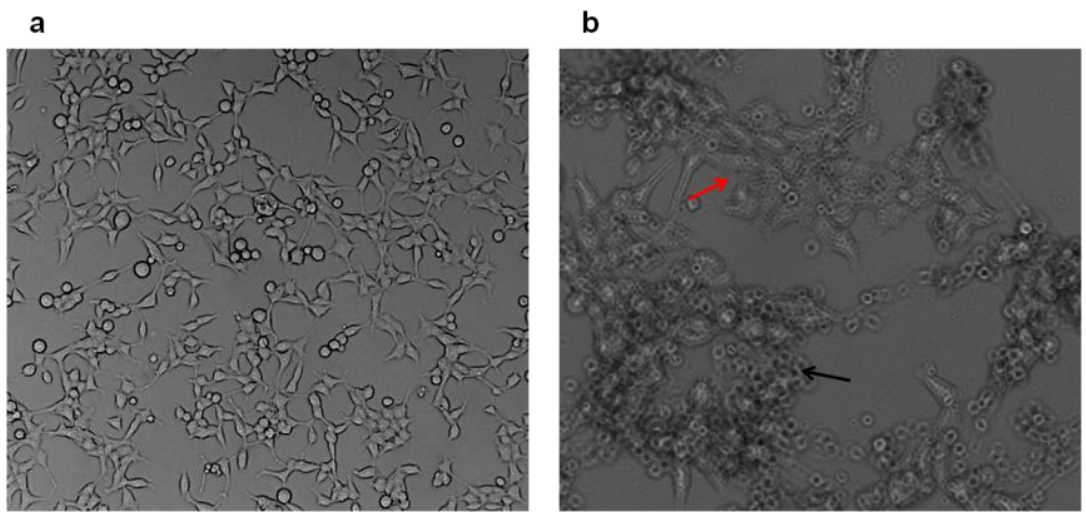

Figure 2. The clones expressing rFSH were isolated by growth under selection pressure with 80-100 ug/ml Hygromycin and 3000-5000 ug/ml G418.

(a) Transfected HEK293 cell lines growth in culture medium without antibiotics. (b)Transfected HEK293 cells growth in culture medium with antibiotics. The red arrow indicates cloned cell lines and the black arrow represents non-cloned cell lines.

Table 1. The ELISA for further screening and identification

\begin{tabular}{lll}
\hline Groups & OD $_{\mathbf{4 5 0}}$ values(means \pm SD) \\
\hline Experimental groups & $0.583 \pm 0.090$ & $0.597 \pm 0.078$ \\
& $0.774 \pm 0.156$ & $0.860 \pm 0.065$ \\
Negative control groups & $0.078 \pm 0.011$ & $0.087 \pm 0.002$ \\
& $0.090 \pm 0.005$ & $0.078 \pm 0.005$ \\
Blank control groups & $0.103 \pm 0.039$ & $0.087 \pm 0.007$ \\
& $0.118 \pm 0.045$ & $0.119 \pm 0.045$ \\
\hline
\end{tabular}

\section{SDS-PAGE Analysis}

After the collection of rFSH from cell culture supernatant, expression of rFSH was determined by $12.0 \%$ sodium dodecyl sulfate-polyacrylamide gel electrophoresis (SDS-PAGE) analysis. The protein migrated on SDS-PAGE with an apparent 
molecular mass of approximately $35 \mathrm{kDa}$ ( red arrow marker), which not consistent with the calculated mass value (30104 Da, 30KD) from the amino acid sequence(as show in Fig. 3).

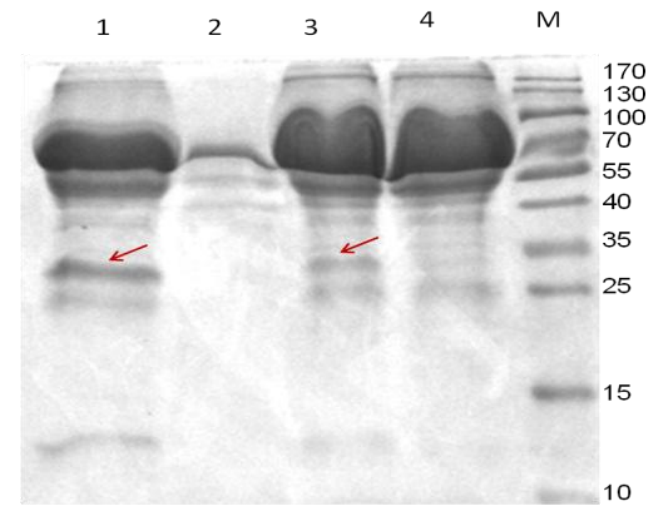

Figure 3. Protein samples were resolved via 12.0\% SDS-PAGE and gels were stained with Coomassie Blue.

Lane 1: concentrated the supematant sample; lane 2:invalid; lane 3: cloned cell lines supernatant sample; lane 4: non-transfected HEK293 cell lines supernatant sample. M: molecular weight marker

\section{Conclusion}

The use of ELISA are necessary to determine the protein productivity of the clones[7-9], and His tags to aid the purification of recombinant proteins have been widely used[10-12]. For obtaining cloned cell lines, we have applied the target gene and a selection marker gene in which the expression vectors. The $\mathrm{rFSH}$ are secreted into the supernatant after posttranslational modifications, we adopted the method of the antibiotics in combination with ELISA and SDS-PAGE to determine. Besides, we are also trying to the purification of the supernatant and simultaneously used of western blot method for further identification, but did not achieved the desired results. In the present study, we have successful constructed the expression vector and established the antibiotics-ELISA screening system for obtaining production cell lines for the production of rFSH with His tags. As for the rFSH biological activities and half-life, we need further assays to verify. Besides, production process of $\mathrm{rFSH}$ are also need to be further optimized.

\section{Acknowledgement}

Financial support for these projects in China (31171731, 31460447, 20142BDH80003, 2013-CXTD002, "555 talent project" of Jiangxi Province, Jiang xi Province Key Laboratory of Bioprocess Engineering and Co-Innovation Center for In Vitro Diagnostic Reagents and Devices of Jiangxi Province ).

\section{References}

[1] Winstel R, Wieland J, Gertz B, et al. Manufacturing of Recombinant Human

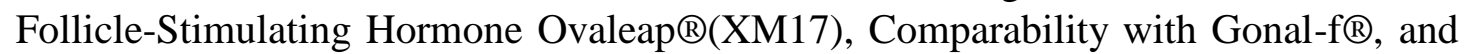
Performance/Consistency[J]. Drugs in R\&D,( 2017) 1-8. 
[2] Sautter K, Enenkel B. Selection of high-producing CHO cells using NPT selection marker with reduced enzyme activity[J]. Biotechnology and bioengineering, (2005)89(5): 530-538

[3] Lee J S, Ha T K, Park J H, et al. Anti-cell death engineering of CHO cells: Co-overexpression of Bcl-2 for apoptosis inhibition, Beclin-1 for autophagy induction[J]. Biotechnology and bioengineering,(2013) 110(8): 2195-2207

[4] DeMaria C T, Cairns V, Schwarz C, et al. Accelerated Clone Selection for Recombinant CHO Cells Using a FACS-Based High-Throughput Screen[J]. Biotechnology progress, (2007)23(2): 465-472.

[5] Kim D J, Seok S H, Baek M W, et al. Highly expressed recombinant human follicle-stimulating hormone from Chinese hamster ovary cells grown in serum-free medium and its effect on induction of folliculogenesis and ovulation[J]. Fertility and sterility,(2010) 93(8): 2652-2660.

[6] Li L, Yu Y, Zhang X, et al. Expression and biochemical characterization of recombinant $\alpha$-l-rhamnosidase $r$-Rhal from Aspergillus niger JMU-TS528[J]. International journal of biological macromolecules, (2016) 85: 391-399.

[7] Sehr P, Zumbach K, Pawlita M. A generic capture ELISA for recombinant proteins fused to glutathione S-transferase: validation for HPV serology[J]. Journal of immunological methods,( 2001) 253(1): 153-162.

[8] oshida M, Hamada T, Amagai M, et al. Enzyme-linked immunosorbent assay using bacterial recombinant proteins of human BP230 as a diagnostic tool for bullous pemphigoid[J]. Journal of dermatological science, (2006) 41(1): 21-30.

[9] Huleatt J W, Nakaar V, Desai P, et al. Potent immunogenicity and efficacy of a universal influenza vaccine candidate comprising a recombinant fusion protein linking influenza M2e to the TLR5 ligand flagellin[J]. Vaccine, (2008) 26(2): 201-214.

[10] Crowe J, Dobeli H, Gentz R, et al. 6xffis-Ni-NTA Chromatography as a Superior Technique in Recombinant Protein Expression/Purification[J]. Protocols for Gene Analysis, (1994): 371-387.

[11] Catanzariti A M, Soboleva T A, Jans D A, et al. An efficient system for high-level expression and easy purification of authentic recombinant proteins[J]. Protein Science,( 2004) 13(5): 1331-1339.

[12] Arnau J, Lauritzen C, Petersen G E, et al. Current strategies for the use of affinity tags and tag removal for the purification of recombinant proteins[J]. Protein expression and purification,( 2006)48(1): 1-13. 